\title{
Extension of Liskov Substitution Principle and Application to Curriculum Management
}

\author{
Raul Sorin Fântână ${ }^{1}$, Nicuşor Minculete ${ }^{2}$, Radu-Emil Precup ${ }^{3}$ \\ ${ }^{1}$ Department of International Business, "Dimitrie Cantemir" Christian University, \\ Str. Bisericii Romane 107, RO-500068 Brasov, Romania \\ E-mail: fantana@universitatea-cantemir.ro \\ ${ }^{2}$ Department of Mathematics and Informatics, Transilvania University of Brasov, \\ Str. Iuliu Maniu 50, RO-500091 Brasov, Romania \\ E-mail: minculete.nicusor@unitbv.ro \\ ${ }^{3}$ Department of Automation and Applied Informatics, Politehnica University of \\ Timisoara, Bd. V. Parvan 2, RO-300223 Timisoara, Romania \\ E-mail: radu.precup@upt.ro
}

\begin{abstract}
This paper offers an extension of the Liskov substitution principle by means of compatible systems, i.e., right or appropriate or functional systems. The compatible systems are represented by compatible sets. The compatible sets are first defined and the new approach to the mathematical modelling of compatible systems is given. The properties of compatible systems are next presented and applied to the curriculum management in higher education. The theoretical results are exemplified by a case study that discusses the curriculum management of two academic programs of study using the information densities of optional, fundamental and specialized courses. A discussion on the systems compatibility in several fields is included and the importance of our multidisciplinary approach to compatible resources management is highlighted.
\end{abstract}

Keywords: compatible sets; compatible systems; curriculum management; information density; Liskov substitution principle; mechatronics

\section{Introduction}

Following the unprecedented development of science and the emergence of interdisciplinary or border branches, specializations in one area can provide explanations and ways to solve the other. Highly contoured borders of some sciences and courses have disappeared. Engineering including mechatronics has multidisciplinary applications in medicine, efficient management forces engineers to have economic knowledge, economists have knowledge of engineering and all students must maximize the outcome of their knowledge. 
Researchers and specializations of academic courses must provide each other knowledge and use specific compatible tools in the curriculum management. The multidisciplinary specialized intellectual resources must be used because of the benefits in terms of value of time and need for excellence.

Since the above aspects have not been proved mathematically, this paper provides the definition of compatible sets by the extension of the Liskov substitution principle (LSP) [1]. This is motivated by numerous examples from different fields that relate in similar terms to elements, sets, activities, which not identical, but compatible insofar as each has a density of identical information. With this regard the main purpose of this paper is to offer a modelling approach that characterizes the compatible systems or sets in the same environment and to apply our approach to academic curriculum management.

This paper uses the symbol $\omega$ to indicate the compatibility relation. In this context, the notation $x \omega y$ outlines that elements $x \in X$ and $y \in Y$ are compatible. As shown in [2], if $A$ and $B$ are independent systems, the problems of the system $A$ are solved using the system $B$ given that:

- both systems contain compatible elements called mirror elements,

- both systems operate in the same environment.

Under these conditions, two or more systems can be compatible. Therefore, the compatibility is defined in [2] as the capacity of two or more systems or components to carry out all tasks as long as they operate in the same environment.

The new idea of this paper stems from the characterization of systems and of their elements given in [2]: if in the system $S_{A}$ represented by the set $A$ all elements are considered known and the elements $x_{\omega y i}$ are unknown, and if in the system $S_{B}$ represented by the set $B$ all elements are considered known including the elements $y_{\omega x i}$, and if the elements $x_{\omega y i}$ are compatible with the elements $y_{\omega x i}$, then, replacing between them the compatible elements or the mirror elements, the system $S_{A}$ will be considered resolved or functional. This means that the system $S_{A}$ is compatible with the system $S_{B}$, and we use the notation $S_{A} \omega S_{B}$.

This paper extends this result by suggesting operations on compatible systems and their characterization by the information density of a system. Programs of study in higher education are targeted.

This paper is organized as follows: the background is presented in the next section. Section 3 is dedicated to the new approach to modelling of compatible systems. The properties of compatible systems are formulated and proved. Section 4 validates the theoretical approach by a case study focused on the curriculum management of two academic programs of study. The conclusions are pointed out in the final section. 


\section{Background}

Many theories on the connection between the performance and the human resource management have been proposed recently [3-5]. Due to the market changes, the schools and universities have become increasingly managerially in their approach [6].

This paper offers a theory and some technical points of view on how to generally keep the freedom, the autonomy and the identity by offering, at the same time, the possibility to co-operate if the fields of activity are compatible. We focus on the academic field as a representative example due to its importance [7-10].

The concept of compatible is often used in the theory of systems of equations. Thus, a system of equations is called compatible if the system has at least one solution. The probability theory states that two events $A$ and $B$ are compatible if they take place in the same sample, namely, if they have at least one common favourable case.

The LSP is very close to compatibility. This principle states that [1] if $A$ is a subtype of $B$, then objects of type $B$ may be replaced with objects of type $A$ (i.e., objects of type $A$ may be substituted by objects of type $B$ ) without altering any of the desirable properties of the environment where $A$ and $B$ exist. As shown in [1], if these conditions are fulfilled in a computer program, the desirable properties of that program (correctness, tasks performed, etc.) are not altered. In addition, the subtypes should fulfil certain constraints.

This paper proposes the need to treat the compatible systems by an extension of the LSP on the basis of observing the common elements of this principle in various fields. The concept of compatible will be used in the sense of right or appropriate or functional. For example, software compatibility is in question when referring to compatible online games; this concerns the ability of a software program to run in a particular operating system. The new contribution of this paper is important because the literature analysis conducted as follows points out the absence of models to characterize all compatible systems. Moreover, our modelling approach can be uses in human resources management and, more general, in compatible resources management. These aspects are advantageous with respect to the state-of-the-art because we bridge the compatibility gap between systems in different fields by a systematic modelling approach.

The electromagnetic compatibility is discussed in $[11,12]$ from the general point of view of the ability of an equipment or system to perform without introducing intolerable disturbances to anything in the same environment; the applications can be related to sensitivity or robustness issues. The compatibility in divergent market systems is studied in [13]; it is proved that the compatibility can be slightly modified if the market is served by fully integrated system suppliers. The compatibility and substitutability of roles in multi-agent systems are analysed in 
[14]; a formal specification of role-based interactions components along with their composition is suggested. The compatibility in mechanics refers to the conditions under which a displacement field can be guaranteed, and compatibility conditions are considered in [15] as particular cases of integrability conditions in the framework of the linear elasticity theory. The compatibility in medicine is related to medical and/or technical subsystems that should operate together in order to achieve several well defined goals with emphasis on robotics [16-21]. The use of innovation related to the compatibility in higher education is analysed in [22] with focus on team-based learning. Several problems related to the Bologna process are treated in $[23,24]$. The need for experiments in control engineering and mechatronics education is pointed out in [8, 25-30].

In the context of the previous section, we consider that an element $x \in X$ is compatible with an element $y \in Y$ if, by replacing $x$ with $y$, the new system $S_{A^{\prime}}$ represented by the set $A^{\prime}=(A \backslash\{x\}) \cup\{y\}$ is functional.

We consider two systems operating in the same environment, namely $S_{A}$ represented by the set $A$ and $S_{B}$ represented by the set $B$, both of them having a finite number of elements that characterize each of the systems:

$$
\begin{aligned}
& A=I \cup X_{\omega y} \cup X, \\
& B=I \cup Y_{\omega x} \cup Y,
\end{aligned}
$$

where the subsets in (1) and (2) are expressed as

$$
\begin{aligned}
& I=\left\{i d_{j} \mid j=1 \ldots m\right\}, X_{\omega y}=\left\{x_{\omega y k} \mid k=1 \ldots p\right\}, X=\left\{x_{i} \mid i=1 \ldots n\right\}, \\
& Y_{\omega y}=\left\{y_{\omega x k} \mid k=1 \ldots p\right\}, Y=\left\{y_{j} \mid j=1 \ldots r\right\}
\end{aligned}
$$

and the subsets $X_{\omega y}$ and $Y_{\omega y}$ fulfil the following properties [2]:

- They have the same number $p$ of elements.

- The elements $x_{\omega y k}$ and $y_{\omega x k}$ are compatible elements or mirror elements, these elements can take the place of, or substitute each other without being identical.

- The identical element $i d_{j} \in I$ is not compatible, and a compatible element is not necessarily an identical element.

- The sets $A$ and $B$ are named compatible if the subset $C_{m s}=C_{m s}(A, B)$ consisting of identical elements, together with the compatible elements (mirror elements), delimits a set of minimum or sufficient characteristics.

- If $x_{i} \in X \cap Y$, then $x_{i}$ is called an identical element. 
An example to illustrate the structure of the two sets $A$ and $B$ is presented in Figure 1.

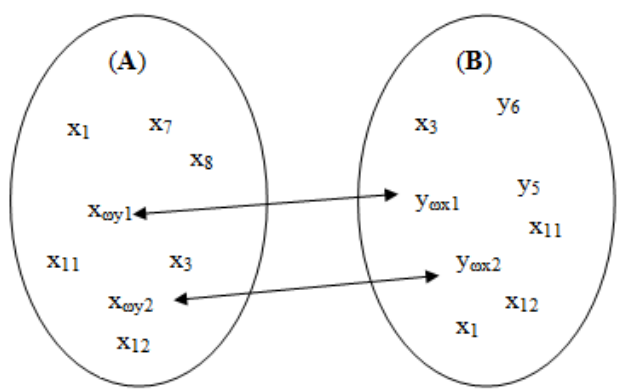

Figure 1

Structure of sets $A$ and $B$

\section{Mathematical Modelling Approach}

The compatible sets $A$ and $B$ are represented by the subset $C_{m s}$ of minimum or sufficient characteristics for which a system $S$ characterised by $C_{m s}$ is functional. The structure of the subset $C_{m s}$ is presented in Figures 2 and 3.

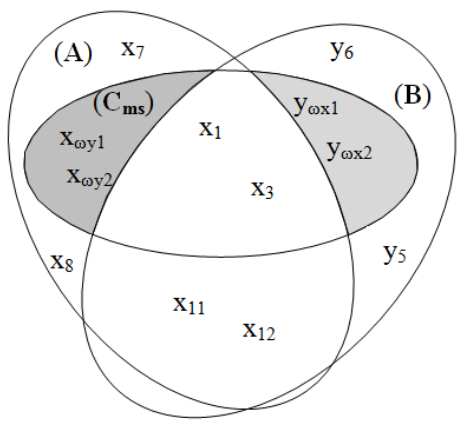

Figure 2

General structure of subset $C_{m s}$

The identical element $x_{i} \in X \cap Y$ is not necessary to the two systems $S_{A}$ and $S_{B}$ to be functional. This is highlighted in Figure 2 by the two elements $x_{11}$ and $x_{12}$. 


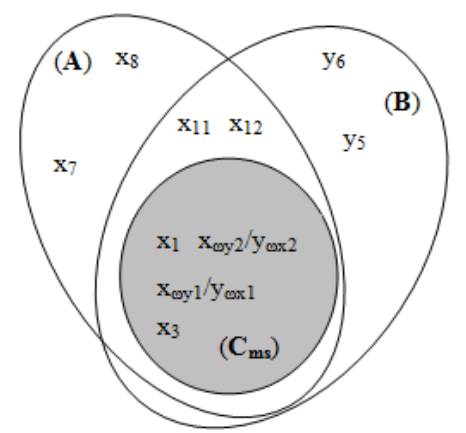

Figure 3

Subset $C_{m s}$ represented as a subset of minimum or sufficient characteristics

The subset $C_{m s}=C_{m s}(A, B)$ is defined as follows [2]: if

$$
X_{A / B} \subseteq X_{\omega y} \cup Y_{\omega x},\left|X_{A / B}\right|=\left|X_{\omega y}\right|=\left|Y_{\omega x}\right|,
$$

then

$$
C_{m s}=C_{m s}(A, B)=I \cup X_{A / B} \cup(X \cap Y),
$$

where $|X|$ is the cardinal of the set $X$.

The additional characteristics $C_{a d}$ :

$$
C_{a d}=(A \cup B) \backslash C_{m s}
$$

do not define, from the point of view of compatibility, the sets $A$ and $B$. This is pointed out by the elements $x_{7}, x_{8}, y_{5}$ and $y_{6}$ in Figure 3 .

As shown in [2], there is a bijective function $f$ that fulfils

$$
\begin{aligned}
& f: B \backslash Y \rightarrow A \backslash X, \\
& f\left(i d_{j}\right)=i d_{j} \text { for }_{i d_{j}} \in I, f\left(y_{\omega x}\right)=x_{\omega y}, y_{\omega x} \in Y_{\omega x} .
\end{aligned}
$$

This function and the inclusion of the identical elements lead to a different representation of Figure 2, shown in Figure 4. Figure 4 highlights both the identical elements $i d_{j}$ and the unnecessary elements $x_{11}$ and $x_{12}$. 


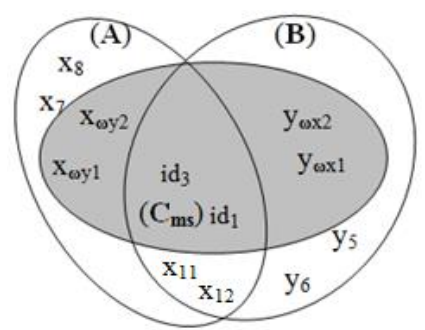

Figure 4

Structure of subset $C_{m s}$ with identical and unnecessary elements included

The properties of the compatibility relation will be presented as follows. These properties are next applied in Section 4.

Proposition 1 (reflexivity). Let the system $S_{A}$ be represented by the set $A$. Then $S_{A} \omega S_{A}$.

Proof. Since the system $S_{A}$ is represented by the set $A$, there is a minimum set of characteristics $C_{m s}(A, A)$ for which the systems $S_{A}$ and $S_{A}$ are compatible, i.e.

$C_{m s}(A, B)=I \cup X_{A / A} \cup X=A$.

Proposition 2 (symmetry). If the relation $S_{A} \omega S_{B}$ holds, then $S_{B} \omega S_{A}$.

Proof. Since the system $S_{A}$ is compatible with the system $S_{B}$, there is a minimum set of characteristics $C_{m s}(A, B)$ for which the systems $S_{A}$ and $S_{B}$ are compatible, i.e.

$C_{m s}(A, B)=I \cup X_{A / B} \cup(X \cap Y)=I \cup X_{B / A} \cup(Y \cap X)=C_{m s}(B, A)$.

Equation (9) indicated that the system $S_{B}$ is compatible with the system $S_{A}$.

Proposition 3 (transitivity). If the relations $S_{A} \omega S_{B}$ and $S_{B} \omega S_{C}$ hold, then $S_{A} \omega S_{C}$.

Proof. Since the system $S_{A}$ is compatible with the system $S_{B}$, there is a minimum set of characteristics $C_{m s}(A, B)$ for which the systems $S_{A}$ and $S_{B}$ are compatible, and equation (5) is applied. Since the system $S_{B}$ is compatible with the system $S_{C}$, there is a minimum set of characteristics $C_{m s}(B, C)$ for which the systems $S_{B}$ and $S_{C}$ are compatible, and the following equation similar to (5) is applied:

$C_{m s}(B, C)=I \cup X_{B / C} \cup(Y \cap Z)$, 
where the set $Z$ is defined similarly to the sets $X$ and $Y$ in (1), (2) and (3), but this set corresponds to $S_{C}$.

Using the definitions of the systems $S_{A}, S_{B}$ and $S_{C}$ represented by the sets $A$, $B$ and $C$, respectively, it follows that there exist a minimum set of characteristics $C_{m s}(A, C)$

$C_{m s}(A, C)=I \cup X_{A / C} \cup(X \cap Z)$,

so the systems $S_{A}$ and $S_{C}$ are compatible.

These three propositions result in the conclusion that the compatibility relation $\omega$ between two systems is an equivalence relation on the set of systems. An equivalence class is given as

$\hat{S}_{A}=\left\{S_{B} \mid S_{A} \omega S_{B}\right\}$.

The antisymmetry property cannot be proved on the compatible sets because these sets also include compatible components that are not identical.

\section{Case Study Concerning Curriculum Management}

Two programs of study $S_{P_{1}}$ and $S_{P_{2}}$, represented by the sets $P_{1}$ and $P_{2}$, are considered in order to exemplify relatively easily the two systems compatibility:

$$
\begin{aligned}
& P_{1}=C_{o} \cup C_{f} \cup C_{s}, \\
& C_{o}=\left\{o c_{i} \mid i=1 \ldots n\right\}, C_{f}=\left\{f c_{j} \mid j=1 \ldots m\right\}, C_{s}=\left\{s c_{k} \mid k=1 \ldots p\right\}, \\
& P_{2}=C_{o}^{*} \cup C_{f} \cup C_{s}^{*}, \\
& C_{o}^{*}=\left\{o c_{i}^{*} \mid i=1 \ldots n^{*}\right\}, C_{f}=\left\{f c_{j} \mid j=1 \ldots m\right\}, C_{s}^{*}=\left\{s c_{k}^{*} \mid k=1 \ldots p\right\},
\end{aligned}
$$

where $o c_{i}$ and $o c_{i}^{*}$ are the optional courses, $f c_{j}$ are the fundamental / imposed courses, $s c_{k}$ and $s c_{k}^{*}$ are the compatible specialized courses, $C_{o}$ and $C_{o}^{*}$ are the sets of optional courses, $C_{f}$ is the set of fundamental courses, $C_{s}$ and $C_{s}^{*}$ are the sets of compatible specialized courses.

Equations (12) and (13) point out that

$$
\left|C_{s}\right|=\left|C_{s}^{*}\right|
$$


namely the two sets of compatible specialized courses have the same number of compatible elements.

We will evaluate the properties of the sets $C_{o}, C_{f}, C_{s}, C_{o}^{*}$ and $C_{s}^{*}$ in a case study related to two academic programs of study, an engineering program $S_{P_{1}}$ and an economic program $S_{P_{2}}$.

$C_{o}$ and $C_{o}^{*}$ may also be empty sets, and they are insignificant sets not as a number but in the subject matter. Examples of insignificant sets or courses for these two programs of study are Sports and Languages.

$C_{f} \neq \varnothing$ for these programs of study. Examples of courses from this set are Statistics, Mathematics and Control Engineering.

Each element $f c_{j} \in C_{f}$ is characterized by the information density $\rho\left(f c_{j}\right)$, with $\rho\left(f c_{j}\right)>\rho_{a c c}(f c), j=1 \ldots m$,

where $\rho_{a c c}(f c)$ is the accepted threshold of density of fundamental courses. The parameter $\rho_{a c c}(f c)$ is fixed.

$C_{s} \neq \varnothing$ and $C_{s}^{*} \neq \varnothing$ for these programs of study. Examples of courses from these sets are Management and Investments.

Each element $s c_{k} \in C_{s}$ is characterized by the information density $\rho\left(s c_{k}\right)$, with

$$
\rho\left(s c_{k}\right)>\rho_{a c c}(s c), k=1 \ldots p,
$$

where $\rho_{a c c}(s c)$ is the accepted threshold of density of specialized courses. The parameter $\rho_{a c c}(s c)$ is also fixed.

The densities can be quantified and measured. They are related as follows to the intersection and union of compatible sets with emphasis on the curriculum management.

If $S_{P_{1}}, S_{P_{2}}, \ldots, S_{P_{q}}$ are compatible programs of study, the intersection of sets containing the compatible (mirror) elements is defined as the set $P_{1} \cap_{\omega} P_{2}$

$P_{1} \cap P_{\omega}=P_{1} \cap P_{2} \cup X_{P_{1} / P_{2}}$,

where

$$
X_{P_{1} / P_{2}}=\left\{s c_{k} \vee s c_{k}^{*} \mid k=1 \ldots p\right\} .
$$


The set $X_{P_{1} / P_{2}}$ allows for the definition of a matrix of programs of study.

The academic curriculum results in three types of professions from the point of view of compatible resources management:

- Profession, which is given by the university diploma or licence or certificate.

- Border profession, built by supplementing a core profession with specific sufficient partial knowledge belonging to other professions. This type represents an effect of relevant rules or of special laws, e.g., mediator, assessor of property, industrial property attorney. The border profession is not a quality of the main profession.

- Specialization, which completes the curricular area by increasing the quality and / or the scope of profession. The specialization is not a profession itself. A specialization course without faculty cannot give equal rights with those conferred by the university diploma.

Therefore, we define the notion of admissible information density $\rho_{a d m}$ as the value under which complementary or mirror elements are not allowed. The admissible information density $\rho_{a d m}$ in higher education can be, for example, the sum of credits of the courses $C_{f}, C_{s}$ and $C_{s}^{*}$. In fact, the compatible elements are some sets (i.e., they represent the information within the programs $S_{P_{1}}$ and $S_{P_{2}}$ in this case study) of a certain information density.

We define the representativity value associated to the program $S_{P_{1}}$ as $V\left(P_{1}\right)$

$$
V\left(P_{1}\right)=\sum_{j=1}^{m} f c_{j}+\sum_{k=1}^{p} s c_{k},
$$

and the representativity value associated to the program $S_{P_{2}}$ as $V\left(P_{2}\right)$

$$
V\left(P_{2}\right)=\sum_{j=1}^{m} f c_{j}+\sum_{k=1}^{p} s c_{k}^{*} .
$$

Thus, for the programs $S_{P_{1}}$ and $S_{P_{2}}$ to be compatible, their representativity values $V\left(P_{1}\right)$ and $V\left(P_{2}\right)$ must fulfil the conditions

$$
\begin{aligned}
& V\left(P_{1}\right)>\rho_{a d m}, \\
& V\left(P_{2}\right)>\rho_{a d m} .
\end{aligned}
$$

If $S_{P_{1}}, S_{P_{2}}, \ldots, S_{P_{q}}$ are compatible programs of study, the union of sets containing the compatible (mirror) elements is defined as the set $P_{1} \cup_{\omega} P_{2}$ 


$$
P_{1} \cup_{\omega} P_{2}=C_{o} \cup C_{o}^{*} \cup C_{f} \cup X_{P_{1} / P_{2}},
$$

The set $P_{1} \cup_{\omega} P_{2}$ in this case study is the mathematical expression of the curriculum. The set $P_{1} \cup_{\omega} P_{2}$ also represents all programs generated by $P_{1}$ and $P_{2}$ to be compatible:

$$
S_{P_{1}} \cup_{\omega} S_{P_{2}}=\hat{S}_{P_{1}}=\hat{S}_{P_{2}} .
$$

The number of programs of study generated by $P_{1}$ and $P_{2}$ is $N\left(P_{1}, P_{2}\right)$

$$
N\left(P_{1}, P_{2}\right)=2^{n+n^{*}}\left(\begin{array}{c}
2 p \\
p
\end{array}\right) \text {. }
$$

As a result, the programs generated by $S_{P_{1}}$ and $S_{P_{2}}$ offer an image on the compatible elements through the courses Management and Investments. If these courses respect in both programs an at least above $\rho_{a d m}$, then these will contain complementary or mirror courses or items. Therefore, the exchange of experts or teachers will be not only acceptable but also useful to those national or international educational systems where the optimisation focusing on the cost minimisation is required. An objective function that can be used with this regard is

$J=\left|C_{o} \cup C_{f}\right|$.

The optimisation problem must be correctly defined and associated with the correct definition of the variables of the objective function $J$ and of the constraints. One such constraint concerns the set $C_{o}$ that belongs to curricular areas and is given by laws.

The profit optimisation can be targeted as well. But this objective involves a different objective function.

Unfortunately, in education, the profit optimisation does not offer a competitive level of preparation, but just a sufficient one. Therefore national bodies involved in decision making in the educational systems should pay special attention to this subject.

The cost optimisation and the profit optimisation lead to minimum and maximum curricular areas. These areas are used in the assessment of the private higher education. However, the cost optimisation and the profit optimisation can be combined in terms of multi-objective optimisation problems. All these optimisation problems must be solved by appropriate algorithms [31-37].

Fuzzy logic can also be involved in our modelling approach [38-44]. The information density values $\rho\left(f c_{j}\right)$ and $\rho\left(s c_{k}\right)$ can be normalised as follows: 


$$
\begin{aligned}
& \rho_{n}\left(f c_{j}\right)=\rho\left(f c_{j}\right) / \sum_{\mathrm{i}=1}^{m} \rho\left(f c_{\mathrm{\imath}}\right), j=1 \ldots m, \\
& \rho_{n}\left(s c_{k}\right)=\rho\left(s c_{k}\right) / \sum_{\tau=1}^{p} \rho\left(s c_{\tau}\right), k=1 \ldots p,
\end{aligned}
$$

where the normalised information densities $\rho_{n}\left(f c_{j}\right)$ and $\rho_{n}\left(s c_{k}\right)$ fulfil the conditions

$$
\begin{aligned}
& 0 \leq \rho_{n}\left(f c_{j}\right) \leq 1, j=1 \ldots m, \\
& 0 \leq \rho_{n}\left(s c_{k}\right) \leq 1, k=1 \ldots p .
\end{aligned}
$$

We define two fuzzy sets. The first fuzzy set is $\left(C_{f}, \mu_{1}\right)$, with the membership function $\mu_{1}$

$$
\begin{aligned}
& \mu_{1}: C_{f} \rightarrow[0,1], \\
& \mu_{1}\left(f c_{j}\right)=\left\{\begin{array}{cc}
\rho_{n}\left(f c_{j}\right), & \text { if } \rho_{n}\left(f c_{j}\right)>\rho_{a c c}(f c), \\
0, & \text { otherwise, }
\end{array} j=1 \ldots m .\right.
\end{aligned}
$$

The second fuzzy set is $\left(C_{s}, \mu_{2}\right)$, where the membership function $\mu_{2}$ is

$$
\begin{aligned}
& \mu_{2}: C_{s} \rightarrow[0,1], \\
& \mu_{2}\left(s c_{k}\right)=\left\{\begin{array}{cc}
\rho_{n}\left(s c_{k}\right), & \text { if } \rho_{n}\left(s c_{k}\right)>\rho_{a c c}(s c), \\
0, & \text { otherwise, }
\end{array} \quad k=1 \ldots p .\right.
\end{aligned}
$$

These two fuzzy sets are an alternative to the modelling of academic curricula. They can be embedded in decision making using the well acknowledged operators specific to fuzzy logic.

\section{Conclusions}

This paper has proposed an extension of the LSP by means of compatible systems. A mathematical modelling approach that involves information densities has been proposed and applied to the curriculum management of two academic programs of study.

Our mathematical modelling approach is presented by means of specific properties of compatible systems and elements including the definition of fuzzy sets. This aspect will be treated as a future research direction by offering a clear modelling algorithm organised in terms of clear steps that should highlight the presence of control systems and mechatronics applications [45-56] in the courses of the engineering programs of study. The generalization to an arbitrary number of programs of study will be treated. 
The future research will be focused on more convincing educational applications and on the analysis and design of compatible control systems. The further extensions of our modelling approach by fuzzy sets will enable the investigation of tools for the analysis and design fuzzy control systems.

\section{Acknowledgement}

This work was supported by a grant from the Romanian National Authority for Scientific Research, CNCS - UEFISCDI, project number PN-II-ID-PCE-2011-30109, by a grant from the Partnerships in priority areas - PN II program of the Romanian National Authority for Scientific Research ANCS, CNDI - UEFISCDI, project number PN-II-PT-PCCA-2011-3.2-0732, and by grants from the from the Partnerships in priority areas - PN II program of the Romanian Ministry of National Education (MEN) - the Executive Agency for Higher Education, Research, Development and Innovation Funding (UEFISCDI), project numbers PN-II-PT-PCCA-2013-4-0544 and PN-II-PT-PCCA-2013-4-0070.

\section{References}

[1] B. H. Liskov, J. M. Wing: A Behavioral Notion of Subtyping, ACM Transactions on Programming Languages and Systems, Vol. 16, No. 6, 1994, pp. 1811-1841

[2] R. S. Fântână: Human Resources Management. A Mathematical Image of The Profession Compatibility. Proceedings of IX ${ }^{\text {th }}$ International Conference on Challenges in Higher Education and Research in $21^{\text {st }}$ Century, Sofia, Bulgaria, 2011, pp. 342-346

[3] P. Fodor, J. Poór: The Impact of the Economic and Financial Crisis on HRM and Knowledge-Management in Hungary and Slovakia - Empirical Research 2008-2009, Acta Polytechnica Hungarica, Vol. 6, No. 3, 2009, pp. 69-91

[4] B. Zemková, J. Talašová: Fuzzy Sets in HR Management, Acta Polytechnica Hungarica, Vol. 8, No. 3, 2011, pp. 113-124

[5] D. E. Guest: Human Resource Management and Performance: Still Searching for Some Answers, Human Resource Management Journal, Vol. 21, No. 1, 2011, pp. 3-13

[6] M. Waring: All in This Together? HRM and the Individualization of the Academic Worker, Higher Education Policy, Vol. 26, 2013, pp. 397-419

[7] F. Farkas, Á. Király: What Makes Higher Education KnowledgeCompatible?, Acta Polytechnica Hungarica, Vol. 6, No. 3, 2009, pp. 93-104

[8] R.-E. Precup, S. Preitl, M.-B. Radac, E. M. Petriu, C.-A. Dragos, J. K. Tar: Experiment-based Teaching in Advanced Control Engineering, IEEE Transactions on Education, Vol. 54, No. 3, 2011, pp. 345-355 
[9] J. Kabók, T. Kis, M. Csüllög, I. Lendák: Data Envelopment Analysis of Higher Education Competitiveness Indices in Europe, Vol. 10, No. 3, 2013, pp. $195-201$

[10] G. Kiss: Teaching Programming in the Higher Education not for Engineering Students, Procedia - Social and Behavioral Sciences, Vol. 103, 2013, pp. 922-927

[11] R. M. Carlton: An Overview of Standards in Electromagnetic Compatibility for Integrated Circuits, Microelectronics Journal, Vol. 35, No. 6, 2004, pp. 487-495

[12] S. Midya, R. Thottappillil: An Overview of Electromagnetic Compatibility Challenges in European Rail Traffic Management System, Transportation Research Part C: Emerging Technologies, Vol. 16, No. 5, 2008, pp. 515534

[13] J.-H. Hahn, S.-H. Kim: Mix-and-Match Compatibility in Asymmetric System Markets, Journal of Institutional and Theoretical Economics, Vol. 168, No. 2, 2012, pp. 311-338

[14] N. Hameurlain: Formalizing Compatibility and Substitutability of Rolebased Interactions Components in Multi-agent Systems, Multi-Agent Systems and Applications IV, M. Pěchouček, P. Petta, L. Z. Varga, Eds., Lecture Notes in Computer Science, Springer-Verlag, Berlin, Heidelberg, Vol. 3690, 2005, pp. 153-162

[15] C. Amrouche, P. G. Ciarlet, L. Gratie, S. Kesavan: On Saint Venant's Compatibility Conditions and Poincaré's Lemma, Comptes Rendus Mathematique, Vol. 342, No. 11, 2006, pp. 887-891

[16] D. C. Klonoff: Designing an Artificial Pancreas System to Be Compatible with Other Medical Devices, Journal of Diabetes Science and Technology, Vol. 2, No. 5, 2008, pp. 741-745

[17] M. J. Hamamura, S. Ha, W. W. Roeck, L. T. Muftuler, D. J. Wagenaar, D. Meier, B. E. Patt, O. Nalcioglu: Development of an MR-Compatible SPECT System (MRSPECT) for Simultaneous Data Acquisition, Physics in Medicine and Biology, Vol. 55, No. 6, 2010, pp. 1563-1575

[18] T. Haidegger, L. Kovács, R.-E. Precup, B. Benyó, Z. Benyó, S. Preitl: Simulation and Control for Telerobots in Space Medicine, Acta Astronautica, Vol. 181, No. 1, 2012, pp. 390-402

[19] W. C. Torres, M. Quintana, H. Pinzón: Differential Diagnosis of Hemorrhagic Fevers Using ARTMAP and an Artificial Immune System, International Journal of Artificial Intelligence, Vol. 11, No. A13, 2013, pp. 150-169

[20] E. G. Christoforou, I. Seimenis, E. Andreou, E. Eracleous, N. V. Tsekos: A Novel, General-Purpose, MR-Compatible, Manually Actuated Robotic 
Manipulation System for Minimally Invasive Interventions Under Direct MRI Guidance, The International Journal of Medical Robotics and Computer Assisted Surgery, Vol. 10, No. 1, 2014, pp. 22-34

[21] H. Nam-Gung, Y.-H. Kim, I.-K. Lim, J.-G. Lee, J.-P. Lee, J.-K. Lee: Assessment of Compatibility between Standard Medical Systems of $\mathrm{u}-$ RPMS and HL7, Ubiquitous Information Technologies and Applications, Y.-S. Jeong, Y.-H. Park, C.-H. Hsu, J. H. Park, Eds., Lecture Notes in Electrical Engineering, Springer-Verlag, Berlin, Heidelberg, Vol. 280, 2014, pp. 387-394

[22] M. Freeman: To Adopt or not to Adopt Innovation: A Case Study of Teambased Learning, The International Journal of Management Education, Vol. 10, No. 3, 2012, pp. 155-168

[23] G. Alimehmeti, X. Hysa: Why the Europeanization of Bologna Process is a Curved road? Trying a Response through the Viable Systems Approach in Reference to Albania, Procedia - Social and Behavioral Sciences, Vol. 47, 2012, pp. 722-728

[24] M. Azzi: The New Pedagogical Practices within the LMD System: Perceptions of EFL Faculty Members, Procedia - Social and Behavioral Sciences, Vol. 69, 2012, pp. 1004-1013

[25] C. Caro, N. Quijano: Low Cost Experiment for Control Systems, Proceedings of 2011 IEEE IX Latin American Robotics Symposium and IEEE Colombian Conference on Automatic Control and Industry Applications (LARC), Bogota, Colombia, 2011, pp. 1-6

[26] M. Gunasekaran, R. Potluri: Low-Cost Undergraduate Control Systems Experiments Using Microcontroller-based Control of a DC Motor, IEEE Transactions on Education, Vol. 55, No. 4, 2012, pp. 508-516

[27] M. Roman, D. Popescu, D. Selişteanu: An Interactive Teaching System for Bond Graph Modeling and Simulation in Bioengineering", Educational Technology \& Society, Vol. 16, No. 4, 2013, pp. 17-31

[28] S. Aleksandrov, Z. Jovanović, D. Antić, S. Nikolić, S. Perić, R. Aleksandrov: Analysis of the Efficiency of Applied Virtual Simulation Models and Real Learning Systems in the Process of Education in Mechatronics, Acta Polytechnica Hungarica, Vol. 10, No. 6, 2013, pp. 5976

[29] M. Atanasijevic-Kunc, V. Logar, M. Papic, J. Bester, R. Karba: Motivation Experiments for Complex Control Systems Education, Proceedings of $10^{\text {th }}$ IFAC Symposium Advances in Control Education (ACE 2013), Sheffield, UK, 2013, pp. 268-273

[30] C. Buiu, A. Buga, A. M. Coman: Teaching Robotics and Virtual Reality in a Synergistic Approach, Proceedings of $7^{\text {th }}$ IEEE International Conference 
on e-Learning in Industrial Electronics (ICELIE 2013), Vienna, Austria, 2013, pp. 71-75

[31] S. Preitl, R.-E. Precup, J. Fodor, B. Bede: Iterative Feedback Tuning in Fuzzy Control Systems. Theory and applications, Acta Polytechnica Hungarica, Vol. 3, No. 3, 2006, pp. 81-96

[32] M.-B. Radac, R.-E. Precup, E. M. Petriu, S. Preitl: Application of IFT and SPSA to Servo System Control, IEEE Transactions on Neural Networks, Vol. 22, No. 12, 2011, pp. 2363-2375

[33] S. Formentin, P. De Filippi, M. Corno, M. Tanelli, S. M. Savaresi: Datadriven Design of Braking Control Systems, IEEE Transactions on Control Systems Technology, Vol. 21, No. 1, 2013, pp. 186-193

[34] M. Shen, W.-N. Chen, J. Zhang, H. S.-H. Chung, O. Kaynak: Optimal Selection of Parameters for Nonuniform Embedding of Chaotic Time Series Using Ant Colony Optimization, IEEE Transactions on Cybernetics, Vol. 43, No. 2, 2013, pp. 790-802

[35] M. Samejima, Y. Saito, M. Akiyoshi, H. Oka: A Predictive Search Method for FAQ-based Question Answering System, International Journal of Artificial Intelligence, Vol. 11, No. A13, 2013, pp. 103-114

[36] L. G. Guzmán, Alix S. Gómez, C. J. Ardila, D. Jabba: Adaptation of the GRASP Algorithm to Solve a Multiobjective Problem Using the Pareto Concept, International Journal of Artificial Intelligence, Vol. 11, No. A13, 2013, pp. 222-236

[37] F. Valdez, P. Melin, O. Castillo: A Survey on Nature-inspired Optimization Algorithms with Fuzzy Logic for Dynamic Parameter Adaptation, Expert Systems with Applications, Vol. 41, No. 14, 2014, pp. 6459-6466

[38] R.-E. Precup, S. Preitl, M. Balas, V. Balas: Fuzzy Controllers for Tire Slip Control in Anti-lock Braking Systems, Proceedings of IEEE International Conference on Fuzzy Systems (FUZZ-IEEE 2004), Budapest, Hungary, 2004, Vol. 3, pp. 1317-1322

[39] R.-E. Precup, S. Preitl: Stability and Sensitivity Analysis of Fuzzy Control Systems. Mechatronics Applications, Acta Polytechnica Hungarica, Vol. 3, No. 1, 2006, pp. 61-76

[40] R.-E. Precup, S. Preitl: PI-fuzzy Controllers for Integral Plants to Ensure Robust Stability, Information Sciences, Vol. 20, No. 20, 2007, pp. 44104429

[41] R.-E. Precup, M. L. Tomescu, S. Preitl: Fuzzy Logic Control System Stability Analysis Based on Lyapunov's Direct Method, International Journal of Computers, Communication \& Control, Vol. 4, No. 4, 2009, pp. 415-426 
[42] Z. C. Johanyák: Fuzzy Modeling of Thermoplastic Composites' Melt Volume Rate, Computing and Informatics, Vol. 32, No 4, 2013, pp. 845857

[43] P. Angelov: Autonomous Learning Systems: From Data Streams to Knowledge in Real-time, John Wiley \& Sons, Chichester, UK, 2013

[44] T. Vollmer, M. Manic, O. Linda: Autonomic Intelligent Cyber-Sensor to Support Industrial Control Network Awareness, IEEE Transactions on Industrial Informatics, Vol. 10, No. 2, 2014, pp. 1647-1658

[45] S. Preitl, R.-E. Precup: On the Algorithmic Design of a Class of Control Systems Based on Providing the Symmetry of Open-loop Bode Plots, Scientific Bulletin of "Politehnica" University of Timisoara, Romania, Transactions on Automatic Control and Computer Science, Vol. 41 (55), No. 1-2, 1996, pp. 47-55

[46] I. Dumitrache, M. Dragoicea: Some Problems of Advanced Mobile Robot Control, Control Engineering and Applied Informatics, Vol. 7, No. 4, 2005 , pp. 11-30

[47] R.-E. Precup, S. Preitl: PI and PID Controllers Tuning for Integral-type Servo Systems to Ensure Robust Stability and Controller Robustness, Electrical Engineering, Vol. 88, No. 2, 2006, pp. 149-156

[48] G. Hermann, G. Benkó, A. Nagy: Retrofitting a Length Measuring Machine for Linear Scale Calibration, Proceedings of $13^{\text {th }}$ International Conference on Intelligent Engineering Systems (INES 2009), Barbados, 2009, pp. 7982

[49] A. Csapó, P. Baranyi: A Unified Terminology for the Structure and Semantics of CogInfoCom Channels, Acta Polytechnica Hungarica, Vol. 9, No. 1, 2012, pp. 85-105

[50] M. Bošnak, D. Matko, S. Blažič: Quadrocopter Control Using an On-board Video System with Off-board Processing, Robotics and Autonomous Systems, Vol. 60, No. 4, 2012, pp. 657-667

[51] J. Vaščák, M. Pal'a: Adaptation of Fuzzy Cognitive Maps for Navigation Purposes by Migration Algorithms, International Journal of Artificial Intelligence, Vol. 8, No. S12, 2012, pp. 20-37

[52] F. G. Filip: A Decision-Making Perspective for Designing and Building Information Systems, International Journal of Computers, Communication \& Control, Vol. 7, No. 2, 2012, pp. 264-272

[53] L. Horváth, I. J. Rudas: Active Knowledge for the Situation-driven Control of Product Definition, Acta Polytechnica Hungarica, Vol. 10, No. 2, 2013, pp. 217-234 
[54] K. Lamár, J. Neszveda: Average Probability of Failure of Aperiodically Operated Devices, Acta Polytechnica Hungarica, Vol. 10. No. 8, 2013, pp. 153-167

[55] D. Gong. S. Liu: A Holographic-based Model for Logistics Resources Integration, Studies in Informatics and Control, Vol. 22, No. 4, 2013, pp. 367-376

[56] L. Kovács, A. Szeles, D. A. Drexler, J. Sápi, I. Harmati, Z. Sápi: Modelbased Angiogenic Inhibition of Tumor Growth using Modern Robust Control Method, Computer Methods and Programs in Biomedicine, Vol. 114, 2014, pp. e98-e110 